Abanico Veterinario. Enero-Diciembre 2020; 10:1-10. http://dx.doi.org/10.21929/abavet2020.28

Artículo Original. Recibido: 18/05/2020. Aceptado: 20/08/2020. Publicado: 26/10/2020. Clave:2020-36.

\title{
Contribución al bienestar animal en la crianza de becerras de reemplazo: alojamiento en pareja
}

\author{
Contribution to animal welfare of dairy calves: housing in pairs
}

\section{Alejos-de la Fuente Isidro1 ID, Almaráz-Buendía Isaac ${ }^{2}$ ID, Peralta-Ortiz Jesús ${ }^{2}$ ID, Meza-Nieto Martín 2 ID, Torres-Cardona Guadalupe ${ }^{\star 2}$ ID}

${ }^{1}$ Universidad Autónoma Chapingo, Departamento de Zootecnia, km 38.5 carr. México - Texcoco, CP 56230, Chapingo, Texcoco, Estado de México, México. ${ }^{2}$ Universidad Autónoma del Estado de Hidalgo, Instituto de Ciencias Agropecuarias, Área Académica de Medicina Veterinaria y Zootecnia, Tulancingo, Hidalgo, CP 43600. *Autor en correspondencia: María Guadalupe Torres Cardona: maria_torres7599@uaeh.edu.mx, Rancho Universitario Ex-Hda. de Aquetzalpa, Av. Universidad km 1, Hidalgo, México A.P. 32 CP.43600. $017717172000 \quad$ ext. 2455. jalejosd@chapingo.mx; isaac_almaraz9974@uaeh.edu.mx; peralta@uaeh.edu.mx; martin_meza3292@uaeh.edu.mx; maria_torres7599@uaeh.edu.mx

\section{RESUMEN}

Con el objetivo de evaluar la respuesta productiva y conductual de becerras Holstein lactantes alojadas en pareja, se utilizaron 30 becerras de una semana de edad hasta el destete ( 8 semanas de edad) y distribuidas aleatoriamente en dos tratamientos: tratamiento 1 becerras alojadas en parejas $(T 1, n=10)$; tratamiento 2 becerras alojadas individualmente $(T 2, n=10)$. No se encontró diferencia significativa ( $p>0.05)$ entre tratamientos en el peso inicial ( $\mathrm{PI}, \mathrm{T} 1=38.18$ vs $\mathrm{T} 2=39.58$, lo que evidencia grupos homogéneos) ni en el consumo total de materia seca (CTMS, T1=56.84 vs T2 $=56.85, \mathrm{~kg}$ ). Sin embargo, las becerras alojadas en pareja ( $\mathrm{T} 1)$ mostraron significativamente mejores resultados en el peso final ( $T 1=75.28$ vs $\mathrm{T} 2=65.74, \mathrm{~kg}$ ), en la ganancia total de peso GTP, $\mathrm{T} 1=37.10 \mathrm{vs} \mathrm{T} 2=26.16, \mathrm{~kg}$ ), en la ganancia diaria de peso (GDP, $\mathrm{T} 1=0.66$ vs $\mathrm{T} 2=0.46, \mathrm{~kg}$ ) y en la conversión alimenticia $(\mathrm{CA}, \mathrm{T} 1=1.53$ vs $\mathrm{T} 2=2.17$ ), respecto a las becerras que se alojaron de manera individual. Se concluye que las becerras que estuvieron alojadas en parejas mostraron mayor respuesta productiva y menor frecuencia de conductas indicadoras de estrés en comparación con las que estuvieron alojadas de manera individual.

Palabras clave: bienestar animal, alojamiento, productividad, comportamiento, becerras Holstein.

\begin{abstract}
To evaluate the productive and behavioral response of pair-housed nursing Holstein calves, 30 calves from one week of age until weaning ( 8 weeks of age) were used and randomly distributed in two treatments: treatment 1 pair-housed calf $(T 1, n=10)$; treatment 2 single-housed calves $(T 2, n=10)$. No significant difference $(p>0.05)$ was found between treatments in initial weight (IP, $T 1=38.18$ vs. $T 2=39.58$, evidencing homogeneous groups) and total dry matter consumption (CTMS, T1=56.84 vs. T2 $=56.85, \mathrm{~kg}$ ). However, calves housed in pairs (T1) showed significantly better results in final weight ( $T 1=75.28 \mathrm{vs}$. $T 2=65.74, \mathrm{~kg})$, total weight gain GTP, $T 1=37.10$ vs. $T 2=26.16, \mathrm{~kg}$ ), daily weight gain (GDP, $T 1=0.66 \mathrm{vs}$. T2=0.46, $\mathrm{kg}$ ) and feed conversion ( $\mathrm{AC}, \mathrm{T} 1=1.53$ vs. $\mathrm{T} 2=2.17$ ), compared to individually housed calves. We concluded that paired calves showed greater productive response and lower frequency of stress-indicative behaviors compared with individually housed calves.
\end{abstract}

Keywords: animal welfare, housing, productivity, behavior, Holstein calves. 


\section{INTRODUCCIÓN}

Bienestar animal se refiere al estado interno de un animal cuando enfrenta al ambiente que lo rodea, por lo que comprende su estado de salud, su percepción del entorno y sus estados mentales (Greiveldinger, 2007). Se ha definido como el estado en que el animal tiene satisfechas sus necesidades fisiológicas básicas, de salud y de comportamiento, frente a los cambios en su ambiente (Broom, 1986); por lo que se percibe cada vez más como un elemento integrante de la calidad global de los alimentos, con implicaciones importantes para la salud animal y la seguridad alimentaria, entre otros.

No obstante, lo anterior, los sistemas intensivos de producción se han diseñado para obtener producciones cada vez mayores a costos cada vez menores, adoptando en muchas ocasiones manejos inadecuados que ocasionan estrés agudo o crónico, dejando de lado las necesidades conductuales de los animales, con lo que se compromete su bienestar; la crianza de becerras no escapa a dicha situación. Actualmente, en la mayoría de las unidades de producción lechera, la becerra recién nacida es criada bajo un esquema que incluye diversas situaciones que le generan estrés, una de las más comunes es alojarlas en corraletas o jaulas individuales como medida de control sanitario, para evitar comportamientos de succión excesiva y para controlar la alimentación; aunque la mayoría de las veces las jaulas son tan pequeñas que restringen e incluso suprimen los patrones de comportamiento individual y social, no permitiendo al animal darse vuelta ni acicalarse la parte posterior del cuerpo, entre otros (Flower y Weary, 2003; Galindo y Orihuela, 2004). El aislamiento de becerras tiene diversos efectos adversos en su bienestar: incrementa la frecuencia de vocalización y la presentación de conductas anormales, aumentando también la respuesta fisiológica al estrés (ritmo cardíaco y los niveles plasmáticos de cortisol), con lo que se afecta la utilización de nutrientes, y por consiguiente, la productividad de los animales; también puede impedir el aprendizaje de habilidades sociales esenciales y necesarias para hacer frente al entorno de grupo al que todo ganado lechero es sometidos más tarde en su vida adulta (Duve y Jansen, 2011; Horvat y Miller-Cushon, 2019).

En un estudio previo, el alojamiento en pares en etapa de lactancia, resultó en animales más relajados, pero la evidencia de mejoría en indicadores productivos no fue concluyente (Chua et al., 2002). Uno de los objetivos principales de la ciencia del bienestar animal, es ofrecer estrategias de manejo que disminuyan el sufrimiento animal, incrementando así su estado de bienestar.

Por lo anterior, el objetivo de esta investigación fue evaluar la respuesta productiva y conductual de becerras Holstein lactantes alojadas en parejas, en comparación con becerras alojadas de manera individual. 


\section{MATERIAL Y MÉTODOS}

\section{Localización}

Esta investigación se realizó en las instalaciones del Rancho Universitario del Instituto de Ciencias Agropecuarias (ICAP) de la Universidad Autónoma del Estado de Hidalgo, ubicado en el municipio de Tulancingo de Bravo, Hidalgo, México. El clima de Tulancingo de Bravo es templado-frío, con una temperatura media anual de $14^{\circ} \mathrm{C}$ y con una precipitación pluvial que oscila entre 500 y $553 \mathrm{~mm}$ por año y se localiza a 2,580 m.s.n.m. (García, 2017).Tulancingo es un valle fértil cuya cuenca lechera produce aproximadamente 40,000 L de leche al día, aportando una cantidad importante al total de producción estatal, con lo que contribuye para que el estado de Hidalgo ocupe el $10^{\circ}$ lugar en la producción nacional de leche de vaca (SIAP, 2018).

\section{Sujetos experimentales y manejo}

Se utilizaron 30 becerras Holstein de una semana de edad. Previo al experimento del día 0 al 7 post nacimiento, todas las becerras recibieron el mismo manejo, el cual consistió principalmente en separarlas de su madre y alojarlas en una corraleta individual donde se les ofreció dos tomas de calostro de $2 \mathrm{~L}$, cada una con un intervalo de $6 \mathrm{~h}$ durante el primer día de nacimiento; a partir del día 2 post nacimiento se alimentaron con $4 \mathrm{~L}$ diarios de leche, dos por la mañana y dos por la tarde (sin rechazo), y desde la primera semana de edad se les ofreció concentrado iniciador comercial y agua a libre acceso.

\section{Tratamientos}

Los animales fueron alojados durante 8 semanas de duración de la prueba en dos tratamientos:

a) Tratamiento 1: becerras alojadas en pareja. A la semana de edad, 20 becerras fueron alojadas en parejas, utilizando 10 corraletas de $2.40 \times 1.25$ metros, diseñadas con un área a la intemperie y otra área cubierta, teniendo diez unidades experimentales.

b) Tratamiento 2: becerras alojadas individualmente. A la semana de edad 10 becerras fueron alojadas en forma individual en corraletas de 1.20 metros x 1.25 metros, diseñadas con un área a la intemperie y otra área cubierta, teniendo diez unidades experimentales.

\section{Variables de Respuesta}

a) Productivas:

i) Peso inicial (PI), Peso final (PF), Ganancia diaria de peso (GDP) y Ganancia total de peso (GTP). Las becerras se pesaron al inicio del experimento y posteriormente cada 15 días. El PI de las becerras se consideró como covariable para confirmar la homogeneidad entre tratamientos en dicha variable y para obtener el PF, GDP y GTP.

ii) Consumo total de materia seca (CTMS). El concentrado iniciador se ofreció a libre acceso, y el consumo se obtuvo por diferencia entre lo 
ofrecido y lo rechazado diariamente. Para obtener el contenido de materia seca del concentrado iniciador y de la leche se utilizó una estufa de aire forzado, hasta que las muestras alcanzaron un peso constante.

iii) Conversión alimenticia (CA). Para calcular la cantidad de alimento necesario para incrementar un $\mathrm{kg}$ de peso vivo en cada becerra, se dividió la cantidad de kilogramos de materia seca consumidos (CTMS) entre la ganancia total de peso (GTP).

\section{b) Conductuales:}

Dos días a la semana se registró el catálogo de conducta de cada becerra, observándolas durante tres horas cada día por ocho semanas; se utilizó un muestreo de barrido en combinación con un registro a intervalos de cinco minutos para los estados, y un muestreo conductual para los eventos (Martin, 2007); obteniendo un total de 576 barridos para cada becerra durante el experimento.

Las variables conductuales consideradas en el catálogo de conducta fueron:

- Estados: parada, echada, caminando, corriendo, comiendo, bebiendo y lamiendo.

- Eventos: vocalizando, orinando y defecando.

Se calculó el porcentaje final de barridos registrados en cada conducta para los estados, y la frecuencia para los eventos.

\section{Análisis estadístico}

Se utilizó un diseño experimental completamente al azar; las variables conductuales se analizaron usando un modelo completamente al azar en medidas repetidas; mientras que las variables productivas se analizaron usando un modelo completamente al azar; en ambas utilizando el procedimiento PROC GLM de SAS (SAS, 2009).

\section{RESULTADOS Y DISCUSIÓN}

En la tabla 1 se muestran las medias de las variables productivas por tratamiento, no encontrando diferencia significativa $(\mathrm{p}>0.05)$ en el $\mathrm{PI}(\mathrm{T} 1=38.18 \mathrm{vs} \mathrm{T} 2=39.58, \mathrm{~kg})$, con lo que se confirma la homogeneidad de las unidades experimentales entre tratamientos respecto a esta variable. El tipo de alojamiento no modificó significativamente ( $p>0.05)$ el consumo total de materia seca; siendo esta variable estadísticamente igual en ambos tratamientos ( $\mathrm{T} 1=56.84$ vs $\mathrm{T} 2=56.85, \mathrm{~kg})$; sin embargo, el tipo de alojamiento modificó significativamente $(p<0.05)$ el peso final, encontrándose un incremento del $14.52 \%$ en las becerras que fueron alojadas en parejas, respecto a las alojadas de manera individual ( $\mathrm{PF}=\mathrm{T} 1: 75.288$ vs T2: $65.747, \mathrm{~kg}$ ). Las variables ganancia total de peso y ganancia diaria de peso también se modificaron por efecto del tipo de alojamiento, observándose incrementos del $41.81 \%$ y $43.04 \%$, respectivamente, en las becerras alojadas en pareja (GTP=T1:37.108 vs T2:26.167, kg; GDP=T1:0.6683 vs T2:0.4672, kg). Respecto a la 
conversión alimenticia, se obtuvieron valores significativamente menores $(p<0.05)$ en $41.83 \%$ en las becerras alojadas en parejas ( $T 1=1.53$ vs $T 2=2.17$ ), lo que significa que este grupo de animales utilizó significativamente menos alimento para alcanzar un kilogramo de ganancia de peso corporal, en comparación con las becerras que se alojaron de manera individual.

Los resultados de esta investigación concuerdan con lo encontrado en otros estudios, donde se reporta que becerras alojadas en compañía de otro u otros individuos de la misma edad, establecen lazos sociales fuertes con sus compañeros, lo cual les permite enfrentar de mejor manera diversas situaciones de estrés; lo que repercute en un menor costo biológico al hacer frente a dichas situaciones estresantes, mostrando una mayor respuesta productiva y una amplia gama de comportamientos (Christensen et al., 2002; Chua et al., 2002; Tapki, 2007; Duve y Jansen, 2011; Jansen y Larsen, 2014;). Se ha encontrado también que terneras alojadas en grupos de 10 o menos, tienen mejores tasas de crecimiento y menores tasas de morbilidad asociadas a enfermedad respiratoria bovina, y con mejores niveles de bienestar (Nabais, 2011; Jansen y Larsen, 2014).

Tabla 1. Variables productivas en becerras Holstein lactantes alojadas en parejas (T1) 0 individualmente (T2)

\begin{tabular}{|c|c|c|c|c|}
\hline \multirow{2}{*}{ VARIABLE } & \multicolumn{2}{|c|}{ Tipos de Alojamiento } & \multirow[b]{2}{*}{ Probabilidad } & \multirow[b]{2}{*}{ C. V. } \\
\hline & $\begin{array}{c}\text { En parejas } \\
\mathrm{T} 1\end{array}$ & $\begin{array}{l}\text { Individual } \\
\text { T2 }\end{array}$ & & \\
\hline Peso inicial (PI, kg) & 38.18 & 39.58 & 0.4946 & 8.79 \\
\hline Peso final (PF, kg) & 75.288 & 65.747 & 0.0153 & 7.95 \\
\hline $\begin{array}{l}\text { Ganancia total de peso } \\
(\text { GTP, kg) }\end{array}$ & 37.108 & 26.167 & 0.0153 & 20.46 \\
\hline $\begin{array}{l}\text { Ganancia diaria de peso } \\
(G D P, k g)\end{array}$ & 0.6683 & 0.4672 & 0.0417 & 24.03 \\
\hline $\begin{array}{l}\text { Consumo total de materia } \\
\text { seca (CTMS, kg) }\end{array}$ & 56.84 & 56.85 & 0.9987 & 11.87 \\
\hline Conversión alimenticia (CA) & 1.53 & 2.17 & 0.0483 & 18.31 \\
\hline
\end{tabular}

T: tratamiento 1: alojadas en pareja; T2: tratamiento 2: alojadas individualmente, C. V.: coeficiente de variación

En la tabla 2 se presentan las medias de las variables conductuales por tratamiento; se encontró que el tipo de alojamiento modificó significativamente $(p<0.05)$ el porcentaje de tiempo que las becerras destinaron a expresar las conductas "parada", "caminando", "bebiendo" y "vocalizando", encontrándose que las becerras que se alojaron en pareja estuvieron $16.26 \%$ menos tiempo paradas ( $\mathrm{T} 1=26.57$ vs $\mathrm{T} 2=31.73$ ), $24.77 \%$ menos 
tiempo caminando ( $\mathrm{T} 1=2.52$ vs $\mathrm{T} 2=3.35), 23.52 \%$ menos tiempo bebiendo ( $\mathrm{T} 1=5.17$ vs $\mathrm{T} 2=6.76$ ) y vocalizaron $41.16 \%$ menos ( $\mathrm{T} 1=2.83$ vs $\mathrm{T} 2=4.81$ ); en comparación con las becerras que estuvieron alojadas de manera individual. Durante el experimento no se observó presencia de enfermedades o conductas anormales en los animales de ambos tratamientos. Los resultados conductuales de esta investigación sugieren que el alojamiento en pareja disminuye la expresión de comportamientos asociados al estrés, como las vocalizaciones. Al respecto De-Paula et al., (2012) indican que en condiciones naturales, las becerras interactúan con otros animales de su misma edad, por lo que el alojamiento individual limita o desaparece esta interacción necesaria.

Tabla 2. Medias de diferentes expresiones conductuales en becerras Holstein lactantes alojadas en parejas (T1) 0 individualmente (T2)

\begin{tabular}{|c|c|c|c|c|}
\hline \multirow[b]{2}{*}{ CONDUCTAS } & \multicolumn{2}{|c|}{ Tipos de alojamiento } & \multirow[b]{2}{*}{ Probabilidad } & \multirow[b]{2}{*}{ C. V. } \\
\hline & $\begin{array}{c}\text { En parejas } \\
\mathrm{T} 1\end{array}$ & $\begin{array}{c}\text { Individual } \\
\text { T2 }\end{array}$ & & \\
\hline \multicolumn{5}{|c|}{ Estados (proporción de tiempo, expresado en porcentaje de barridos) } \\
\hline Parada & 26.57 & 31.73 & 0.0280 & 5.2 \\
\hline Echada & 43.33 & 45.18 & 0.5152 & 3.6 \\
\hline Caminando & 2.52 & 3.35 & 0.0464 & 8.7 \\
\hline Bebiendo & 5.17 & 6.76 & 0.0020 & 4.2 \\
\hline Comiendo & 5.48 & 4.07 & 0.1547 & 5.1 \\
\hline Lamiendo & 6.58 & 7.25 & 0.4438 & 2.2 \\
\hline \multicolumn{5}{|l|}{ Eventos (frecuencias) } \\
\hline Vocalizando & 2.83 & 4.81 & 0.0291 & 3.6 \\
\hline Orinando & 2.08 & 2.98 & 0.7515 & 3.1 \\
\hline Defecando & 0.68 & 0.58 & 0.3958 & 2.2 \\
\hline
\end{tabular}

T1: tratamiento 1: alojadas en pareja; T2: tratamiento 2: alojadas individualmente, C. V.: coeficiente de variación

Los métodos de alojamiento de becerras tienen un impacto importante en la salud y potencialidad del animal en su futura producción (Quigley, 2001); Se cree que el alojamiento individual ayuda a controlar enfermedades, comportamiento anormal de succión y el consumo de alimento. Si bien se ha propuesto que el alojamiento individual simplifica la detección de enfermedades y el manejo en general de las becerras, la práctica ha sido altamente criticada por el hecho de que se limita el grado en el que la becerra puede comportarse naturalmente (Nabais, 2011; Jansen y Larsen, 2014).

El aislamiento social es estresante para las becerras y puede impedir el aprendizaje de habilidades sociales esenciales y necesarias para hacer frente al ambiente de grupo al que todas las vacas lecheras son sometidos en su vida adulta (Morisse et al., 2000; Quigley, 2001; Horvat y Miller-Cushon, 2019). Las becerras alojadas individualmente presentan alteraciones de comportamiento, como permanecer de pie por periodos largos, caminar de forma más insegura, son reacias a cambiar de postura, y al crecer desarrollan estereotipias motivadas por falta de actividad social. 
El aislamiento en el pre destete también podría repercutir en la adaptación al alojamiento en grupo después del destete, especialmente si lo hacen con animales que habían estado en grupo durante el periodo de lactancia (Morisse et al., 2000; Quigley, 2001; Mandel et al., 2016). En contraste, las becerras alojadas en grupo son más seguras (Nabais, 2011), y manifiestan un mayor repertorio de actividades propias de la especie, entre ellas la expresión de comportamientos sociales, como el juego y el aprendizaje desde sus congéneres (Duve y Jansen, 2012; Costa et al., 2016). Las interacciones sociales pueden ayudarles a hacer frente a situaciones de estrés. Las becerras alojadas en grupos o en parejas están menos estresadas durante la manipulación y traslado y son más rápidas para iniciar el consumo de alimento sólido después del destete, en comparación con las alojadas individualmente; lo que indica que son más capaces de hacer frente a la eliminación de la alimentación líquida (Cobb et al., 2014).

El sistema de alojamiento en grupos pequeños o en parejas puede considerarse viable y económico en términos de salud, desarrollo y comportamiento (Lakshman et al., 2009; Krachun y De-Pasillé, 2010; Faerevik et al., 2010; Duve y Jansen, 2011). Aún cuando la calidad del alojamiento puede que no mejore la nutrición y el manejo cuando estos sean deficientes, sí puede arruinar la efectividad de un excelente programa de manejo y alimentación. Aquí radica la importancia del estudio de la conducta de becerras en relación con los sistemas de alojamiento (Morisse et al., 2000; Costa et al., 2016). Lo anterior se constató en el presente estudio, donde bajo un mismo esquema de alimentación y con consumos similares de leche y concentrado, los animales alojados en pareja mostraron una conducta más calmada y con mejor repertorio de conductas propias de la especie. Este mejor desempeño conductual se reflejó en una mejor conversión alimenticia, probablemente por efecto de un mejor uso de los nutrientes. La diferencia en la mejor conversión alimenticia representa un mayor beneficio económico con el uso de este sistema.

Un último punto de importancia para el futuro de las unidades de producción animal es el "costo ético" en la producción (Capó, 2005); si el costo ético es alto, significa que los animales son pobremente tratados y su bienestar es bajo; aquellas unidades de producción en que las prácticas de manejo tradicionales persistan en producir sufrimiento innecesario a los animales, serán menos competitivas en el mercado. El cambio tiene que ver, no solo con que los animales deben presentar ausencia de sufrimiento, sino que deben experimentar placer, disfrute (comportamientos de juego, la comida de buena calidad nutritiva/sensorial, el sexo y el contacto social), el cual es indispensable para brindar un bienestar animal completo (Balcombe, 2009). 


\section{CONCLUSIONES}

Bajo las condiciones en que esta investigación fue realizada, con el objetivo de comparar los sistemas de alojamiento en becerras Holstein, los resultados encontrados sugieren que el alojamiento en parejas es más apropiado, en comparación al alojamiento individual, ya que incrementa la respuesta productiva y disminuye la expresión de los patrones conductuales, indicadores de estrés como las vocalizaciones; por lo que es posible concluir que el alojamiento en pareja mejora el bienestar de las becerras.

\section{AGRADECIMIENTOS}

Los autores desean agradecer el apoyo brindado para la realización de este proyecto al Programa de Mejoramiento del Profesorado (PROMEP/103.5/08/5165).

\section{LITERATURA CITADA}

BALCOMBE J. 2009. Animal pleasure and its moral significance. Appl Anim Behav Sci; 118(3-4):208-216. ISSN:0168-1591. https://doi.org/10.1016/j.applanim.2009.02.012

BROOM DM. 1986. Effects of group-rearing or partial isolation on later social behavior of calves. Anim Behav; 26:1255-1263. ISSN: 0003-3472. https://doi:10.1016/00033472(78)90116-1

CAPÓ MM. 2005. Aplicación de la bioética al bienestar y al derecho de los animales. $1^{\text {a }}$ Edición. Ed. Complutense. España. ISBN: 84-7491-771-9.

CHRISTENSEN JW, Ladewing J, Sondergaard E, Malmkvist J. 2002. Effects of individual versus group stabling on social behavior in domestic stallion. Appl Anim Behav Sci; 75(3):233-248. ISSN:0168-1591. https://doi.org/10.1016/S0168-1591(01)00196-4

CHUA B, Coenen JE, Denle V, Weary DM. 2002. Effects of pair versus individual housing on the behavior and performance of dairy calves. J. Dairy Sci; 85(2):360-364. ISSN: 00220302. https://doi:10.3168/jds.s0022-0302(02)74082-4

COBB CJ, Obeidat BS, Sellers MD, Pepper-Yowell AR, Hanson DL, Ballou MA. 2014. Improved performance and heightened neutrophil responses during the neonatal and weaning periods among outdoor group-housed Holstein calves. J. Dairy Sci; 97(2):930939. ISSN: 0022-0302. https://doi:10.3168/jds.2013-6905

COSTA JHC, Von Keyserlingk MAG, Weary DM. 2016. Invited review: Effects of group housing of dairy calves on behavior, cognition, performance, and health. J. Dairy Sci; 99(4):2453-2467. ISSN: 0022-0302. https://doi.org/10.3168/jds.2015-10144 
DE-PAULA V, De-Passillé AM, Weary DM. 2012. Effects of the early social environment on behavioral responses of dairy calves to novel events. J. Dairy Sci; 95:5149-5155. ISSN: 0022-0302. https://doi.org/10.3168/jds.2011-5073

DUVE OR, Jansen MB. 2011. The level of social contact affects social behavior in preweaned dairy calves. Appl Anim Behav Sci; 135:34-43. ISSN:0168-1591. https://doi.org/10.1016/j.applanim.2011.08.014

DUVE LR, Jensen MB. 2012. Social behavior of young dairy calves housed with limited or full social contact with a peer. J. Dairy Sci; 95:5936-5945. ISSN: 0022-0302. https://doi.org/10.3168/jds.2012-5428

FAEREVIK G, Jensen MB, Boe KE. 2010. The effect of group composition and age on social behavior and competition in groups of weaned dairy calves. J. Dairy Sci; 93:42744279. ISSN: 0022-0302. https://doi.org/10.3168/jds.2010-3147

FLOWER FC, Weary DM. 2003. The effects of early separation on the dairy cow and calf. Anim. Welfare;12:339-348. ISSN:0962-7286.

https://www.ingentaconnect.com/content/ufaw/aw/2003/00000012/00000003/art00004

GALINDO MFA, Orihuela TA. 2004. Etología Aplicada. 1a. Edición. México. Universidad Nacional Autónoma de México. México. ISBN: 970-32-1471-1

GARCÍA Enriqueta. 2017. Modificaciones al Sistema de Clasificación Climática de Köppen. 5a Edición. Serie Libros UNAM. México. ISBN-10 (02): 970-32-1010-4

GREIVELDINGER I. 2007. Emotional experience in sheep: Predictability of a sudden event lowers subsequent emotional responses. $J$ of Physiology and Behaviour; 92:675683. ISSN 0031-9384. https://doi.org/10.1016/j.physbeh.2007.05.012

HORVATH, K. C., \& Miller-Cushon, E. K. (2019). Evaluating effects of providing hay on behavioral development and performance of group-housed dairy calves. J. Dairy Sci; 102(11): 10411-10422 ISSN: 0022-0302. https://doi.org/1010.3168/jds.2019-16533

JENSEN MB, Larsen LE. 2014. Effects of level of social contact on dairy calf behavior and health. J. Dairy Sci; 97(8):5035-44. ISSN: 0022-0302. https://doi.org/10.3168/jds.20137311

KRACHUN CR, De-Passillé AM. 2010. Play behaviour in dairy calves is reduced by weanin and by a low energy intake. Appl Anim Behav Sci; 122(2-4):71-76. ISSN:01681591. https://doi.org/10.1016/j.applanim.2009.12.002 
LAKSHMAN KB, Harnarain P, Ramesh CP, Artabandhu S. 2009. Hemato-biochemical changes, disease incidence and live weight gain in individual versus group reared calves fed on different levels of milk and skim milk. Anim Sci J; 80:149-156. ISSN:1740-0929. https://doi.org/10.1111/j.1740-0929.2008.00620.x

MANDEL R, Whay HR, Klement E, Nicol CJ. 2016. Invited review: Environmental enrichment of dairy cows and calves in indoor housing. J. Dairy Sci; 99 (3):1695-1715. ISSN: 0022-0302. https://doi.org/10.3168/jds.2015-9875

MARTIN PMB. 2007. Measuring behavior: An introductory guide. 3rd. Edition. Cambridge. Cambridge University Press. ISBN-13: 978-0521535632. ISBN-10: 0521535638.

MORISSE JPD, Huonnic D, Cotte JP, Martrenchar A. 2000. The Effect of Four Brous Feed Supplementations On Different Welfare Traits In Veal Calves. Anim Feed Sci Tech; 84:129-136. ISSN: 0377-8401. https://doi.org/10.1016/S0377-8401(00)00112-7

NABAIS UAR. 2011. Medição de proteínas séricas e imunoglobulinas como indicador da transferencia de imunidade passiva em vitelos. Teses de Mestrado. Universidade Técnica de Lisboa, Lisboa.

https://www.repository.utl.pt/bitstream/10400.5/3569/1/Medi\%C3\%A7ao\%20de\%20prote\%C3\% ADnas\%20s\%C3\%A9ricas\%20e\%20imunoglobulinas\%20como\%20indicador\%20da\%20transfer encia\%20de\%20imunidade\%20passiva\%20em\%20vitelos.pdf

QUIGLEY J. 2001. Calf Note \# 64 -Métodos de alojamiento: influyen en el comportamiento del ternero? Ficha técnica. Recuperado el 29 de enero 2019: http://www.calfnotes.com

SAS (Statistical Analysis System). 2009. User's Guide: Statistics, Version 9.02. Edition SAS Inst., Inc. Cary. N. C.

SIAP. Servicio de información agroalimentaria y pesquera. 2018. Secretaría de agricultura, ganadería, desarrollo rural, pesca y alimentación. México. https://www.gob.mx/siap

TAPKI I. 2007. Effects of individual or combined housing systems on behavioural and growth responses of dairy calves. Acta Agr Scand A-AN; 57:55-60. ISSN:0906-4702; EISSN:1651-1972. https://doi.org/10.1080/09064700701464405 